\title{
Trains of attosecond pulses structured with time-ordered polarization states
}

\author{
Laura Rego, * (i) Julio San Román, (i) Luis Plaja, (i) and Carlos Hernández-García (i) \\ Grupo de Investigación en Aplicaciones del Láser y Fotónica, Departamento de Física Aplicada, University of Salamanca, E-37008 Salamanca, \\ Spain \\ *Corresponding author: laura.rego@usal.es
}

Received 17 August 2020; revised 4 September 2020; accepted 5 September 2020; posted 8 September 2020 (Doc. ID 404402); published 6 October 2020

\begin{abstract}
Ultrafast laser pulses generated at the attosecond timescale represent a unique tool to explore the fastest dynamics in matter. An accurate control of their properties, such as polarization, is fundamental to shape three-dimensional laser-driven dynamics. We introduce a technique to generate attosecond pulse trains whose polarization state varies from pulse to pulse. This is accomplished by driving high-harmonic generation with two time-delayed bichromatic counter-rotating fields with proper orbital angular momentum (OAM) content. Our simulations show that the evolution of the polarization state along the train can be controlled via OAM, pulse duration, and time delay of the driving fields. We, thus, introduce an additional control into structured attosecond pulses that provides an alternative route to explore ultrafast dynamics with potential applications in chiral and magnetic materials. (๑) 2020 Optical Society of America
\end{abstract}

https://doi.org/10.1364/OL.404402

The most fundamental mechanisms in atoms, molecules, or solid systems are governed by dynamic interactions occurring at the attosecond timescale. The quest of obtaining light pulses at such short timescales, in order to drive, prove, and observe such dynamics, has driven the scientific community's efforts towards the development of coherent high-frequency light sources during the last two decades. As a consequence of the uncertainty principle, ultrashort pulses must contain coherent radiation spanning into the extreme-ultraviolet (XUV) or x-ray regimes in order to reach the sub-femtosecond timescale. While recently it has been shown that high-energy sub-femtosecond pulses can be obtained from free-electron lasers, highly coherent light bursts emitted at the attosecond timescale can be obtained through high-order harmonic generation (HHG). Shortly after the first demonstration of the comb of high-order harmonics driven by intense lasers [1,2], Farkas and Tóth theoretically forewarned of their characteristic sub-femtosecond duration [3]. Since its experimental confirmation by several groups in the early 2000s [4,5], HHG stands as the most robust tool not only to generate pulses down to few tens of attoseconds, but also to gain control on the properties of XUV radiation with unprecedented accuracy. Attosecond pulse trains provide extraordinary possibilities as quantum stroboscopes [6] for the time-control electron dynamics and the imaging of the electron's quantum state.

The success of HHG to produce attosecond pulses resides in its extraordinary coherence. At the microscopic level, the process is well understood from a semiclassical approach [7]. First, the driving laser field ionizes an electronic wavepacket, which is subsequently steered away from its parent ion. When the driving field reverses its sign, the wavepacket is driven back to the parent ion. Upon recombination, the kinetic energy acquired during the wavepacket excursion is released as high-order harmonics of the driving laser. This sub-femtosecond process is repeated at each half-cycle of the driving pulse, thus leading to the generation of a train of attosecond pulses.

One of the properties of HHG attosecond pulses whose control has been elusive for many years is spin angular momentum (SAM), associated with polarization. The generation of high harmonics and attosecond pulses with different polarization states is of great interest as a tool to probe the fastest spin dynamics in materials, and to uncover fundamental understanding on spin scattering and transport on attosecond timescales. Until recently, the dynamics leading to HHG were restricted along the direction of the linear polarization of the driving field, thus limiting the harmonic photons to linearly polarized states. If driven elliptically, the recombination probability diminishes, drastically reducing the yield of the high-order harmonic emission [8]. Fortunately, different techniques have recently exploited the extremely coherent nature of HHG to generate high-order harmonics-and attosecond pulses-with polarization states from linear to circular [9]. The use of non-symmetric targets, such as molecules or solids $[10,11]$, or the use of proper combinations of linearly polarized drivers with different frequencies [12] enable the generation of elliptically polarized harmonics. Other approaches made use of proper macroscopic configurations, such as the use of non-collinear counter-rotating driving beams with the same color [13] — which allowed for the production of circularly polarized attosecond pulses for the first time $[14,15]$ —or the combination of two HHG sources [16,17].

The first technique that allowed the generation of circularly polarized high-order harmonics used a driving field composed of two-color, counter-rotating, circularly polarized laser fieldsthe so-called bi-circular driver [18-20], which can present 
high ionization and recombination rates [21]. This technique stands nowadays as an efficient method to generate circularly polarized harmonics with brightness and energies comparable to single-color linearly polarized HHG $[22,23]$. The primary spectral feature of bi-circular HHG is the generation of pairs of circularly polarized harmonics with opposite helicity-right circular (RCP) and left-circular (LCP)—due to SAM conservation. The generation of both LCP and RCP harmonics from bi-circular HHG driving results in the emission of linearly polarized attosecond trains of pulses, where the polarization tilt angle rotates from pulse to pulse.

Recently, the introduction of orbital angular momentum (OAM) in the driving pulses, has allowed us to not only to generate circularly polarized harmonic vortex beams, but also to control the polarization of the attosecond pulse trains in the bi-circular scheme [24]. However, up to now, SAM-or the polarization state- has been introduced at the attosecond timescale as a time-independent variable. To the best of our knowledge, there is no proposal-nor experimental demonstration - yet to produce attosecond pulse trains with controlled, well resolved, time-ordered polarization. We note, however, that some recent works have reported the generation of high harmonics whose polarization state slightly changes along the course of the pulse as a result of the highly nonlinear nature of HHG $[11,25]$.

In this Letter, we introduce a technique to generate trains of attosecond pulses with different — and controlled—polarization states from pulse to pulse, thus presenting time-ordered SAM along the pulse train. The control knob lies on the proper choice of the combination of two time-delayed bi-circular driving fields, with different OAM content. Very recently, HHG has been proven to introduce time-dependent OAM into a light beam, denoted as self-torque [26]. In this work, we demonstrate that driving HHG with two time-delayed bi-circular vortex beams with properly chosen OAM content results in trains of attosecond pulses of the SAM state changing sequentially from pulse to pulse. Similar schemes that make use of time-delayed pulses with different SAM, instead of OAM, are now widely used, such as the polarization gating technique [27].

It is already known that introducing OAM in bi-circular driving results in high-order harmonics with opposite helicity, that can be spatially separated in the far-field into a double ring profile [24], as we schematically illustrate in Fig. 1(a). If we consider an OAM bi-circular field composed of two pulses with fundamental $\left(\omega_{1}\right)$ and doubled frequency $\left(\omega_{2}=2 \omega_{1}\right)$, counterrotating $\left(\sigma_{1}=-1 \mathrm{LCP}, \sigma_{2}=1 \mathrm{RCP}\right)$ and with topological charge - or OAM content—of $\ell_{1}$ and $\ell_{2}$, respectively, the difference in divergence in the far-field between the LCP and RCP harmonics is given by $\Delta \beta \propto\left(2 \ell_{1}-\ell_{2}\right)\left|\ell_{1}+\ell_{2}\right| /\left(\ell_{1}+\ell_{2}\right)$ [24]. As a general trend, the spatial separation between the LCP and RCP harmonic rings increases for larger differences between $\ell_{1}$ and $\ell_{2}$, if $\ell_{1}$ and $\ell_{2}$ have opposite signs. The outer (inner) ring-with larger (smaller) divergence- exhibits the same helicity as that of the driving pulse with higher (lower) $|\ell|$. For the convention adopted in this work, if $\left|\ell_{1}\right|>\left|\ell_{2}\right|$, the far-field outer ring is composed of LCP harmonics, whereas if $\ell_{2}|>| \ell_{1} \mid$, it is composed of RCP harmonics, as illustrated in Fig. 1(a). From the temporal point of view, by selecting the high-order harmonics emitted at each of the rings, attosecond pulse trains with LCP or RCP states are generated with a time-independent polarization state. (a)
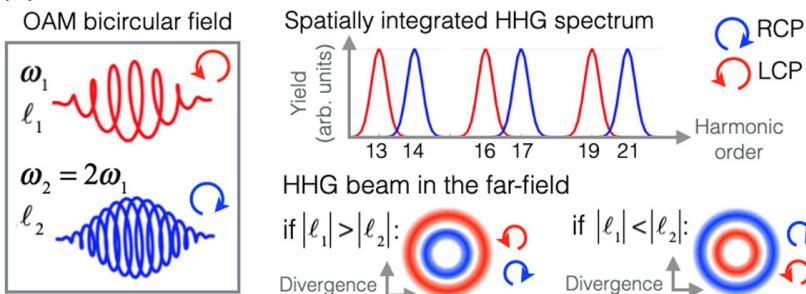

HHG beam in the far-field

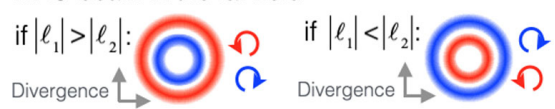

(b)

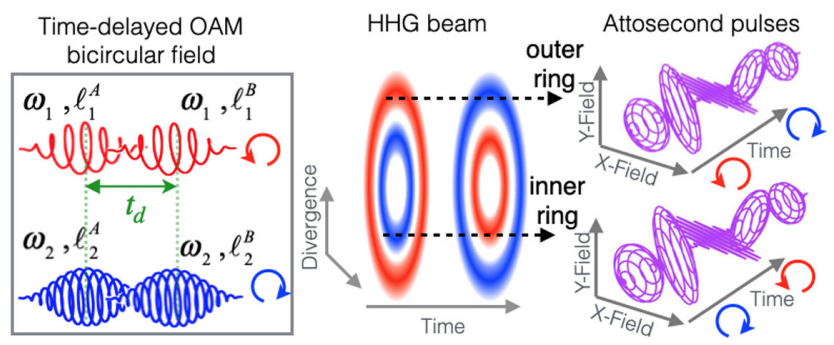

Fig. 1. (a) Scheme of polarization control of HHG driven by an OAM bi-circular field $\left(\omega_{1}, \ell_{1}, \mathrm{LCP} ; 2 \omega_{1}, \ell_{2}, \mathrm{RCP}\right)$. The HHG spectrum is composed of pairs of circularly polarized harmonics with opposite helicity, separated in divergence in the far-field. (b) Generation scheme of trains of attosecond pulses with time-ordered polarization states. In this case, HHG is driven by two time-delayed OAM bi-circular fields, $A$ and $B$, where, for this example, we have chosen $\left|\ell_{1}^{A}\right|>\left|\ell_{2}^{A}\right|$ and $\left|\ell_{1}^{B}\right|<\left|\ell_{2}^{B}\right|$. As a consequence, in the outer (inner) ring, the polarization of the harmonics - and the attosecond pulses - varies continuously in time from LCP (RCP) to RCP (LCP), as shown schematically in the right panel.

In order to introduce a temporal dependency into the polarization state of the attosecond pulses within the train, we drive HHG with a combination of two time-delayed OAM bicircular beams with opposite topological charges [see Fig. 1(b)]. If we denote each of the OAM bi-circular beams as $A$ and $B$, we require an OAM combination of $\left|\ell_{1}^{A}\right|=\left|\ell_{2}^{B}\right|$ and $\left|\ell_{1}^{B}\right|=\left|\ell_{2}^{A}\right|$, maintaining $\ell_{1}$ and $\ell_{2}$ with opposite signs, as mentioned before. The temporal delay between the two OAM bi-circular beams, $t_{d}$, causes the emitted high-order harmonics in each of the rings to evolve from LCP (RCP) to RCP (LCP), as depicted schematically in Fig. 1(b). For this figure, we have selected $\left|\ell_{1}^{A}\right|>\left|\ell_{2}^{A}\right|$ and $\left|\ell_{1}^{B}\right|<\left|\ell_{2}^{B}\right|$, and, thus, the polarization state of the emitted attosecond pulse trains will evolve from LCP (RCP) to RCP (LCP) at the outer (inner) harmonic ring. The pace of SAM changes can be controlled through the OAM of the driving pulses and their temporal properties (pulse duration and time delay).

We have performed simulations of macroscopic HHG in an argon gas jet, including propagation. Our calculations combine the strong-field approximation with the electromagneticfield propagator [28]. This theoretical approach successfully describes the macroscopic HHG in several configurations involving SAM and/or OAM [13-16,24,26]. We model the driving pulses with a $\sin ^{2}$ envelope, with central wavelengths of $800 \mathrm{~nm}(400 \mathrm{~nm})$ for the $\omega_{1}\left(2 \omega_{1}\right)$ driver and a peak intensity of $1.4 \times 10^{14} \mathrm{~W} / \mathrm{cm}^{2}$ each. The spatial structure of the laser beams is represented as a Laguerre-Gaussian beam propagating in the $z$ direction, with topological charge $\ell$ and radial nodes index $p$. The beam waist, $w_{0}$, of each laser beam is chosen so 
(a)

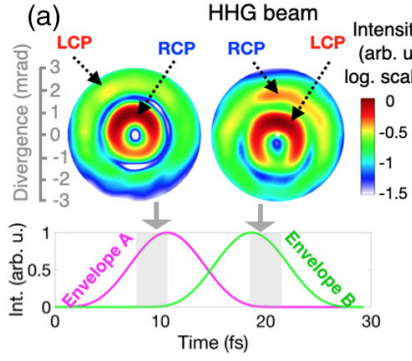

(d)

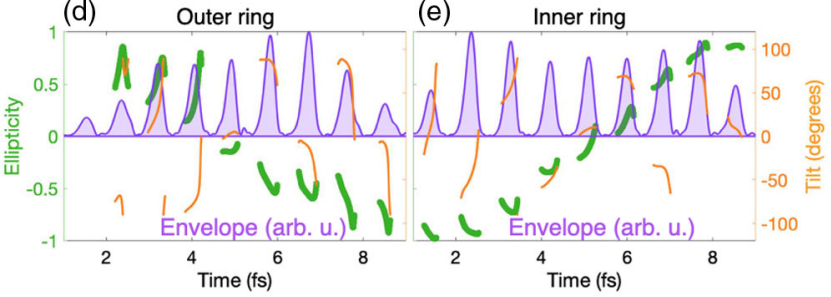

Fig. 2. (a) Spatial intensity profile of the HHG beam obtained with an OAM bi-circular field with $\ell_{1}^{A}=3, \ell_{2}^{A}=-2$ (and, thus, $\ell_{1}^{B}=2$, $\left.\ell_{2}^{B}=-3\right)$. The helicity of the inner and outer rings changes along time. In panels (b) and (c), we show the HHG spectrum of the outer and inner rings, whereas in panels (d) and (e) we show the corresponding attosecond pulse train envelopes (purple), ellipticity (green), and tilt angle (orange).

that the different OAM modes overlap at the rings of maximum intensity.

In Fig. 2, we present the results of the simulations when considering $\ell_{1}^{A}=3, \ell_{2}^{A}=-2$ (and thus $\ell_{1}^{B}=2, \ell_{2}^{B}=-3$ ), all of them with $p=0$. The pulse duration of each driver is $7.7 \mathrm{fs}$ in full width at half-maximum (FWHM) in intensity, and the temporal delay between the $A$ and $B$ drivers is $t_{d}=7.7 \mathrm{fs}$. The waist of the driving beams, $w_{0}$, is $30 \mu \mathrm{m}$ for $\ell= \pm 2$ and $24.54 \mu \mathrm{m}$ for $\ell= \pm 3$. Looking at the spatial intensity profile of the HHG beam in Fig. 2(a), integrated over harmonic orders above the 10th, we distinguish two spatial rings. In Figs. 2(b) and 2(c), we show the far-field HHG spectrum of the outer ring (detected at $1.6 \mathrm{mrad}$ ) and that at the inner ring (detected at $0.6 \mathrm{mrad}$ ), respectively. In both HHG spectra, we can already distinguish the pairs of LCP (red line) and RCP (blue line) harmonics, a well-known result of SAM conservation in HHG driven by the bi-circular field [22]. Note that we observe a frequency shift in the harmonics, which is induced by the pulse envelope through the time-dependent variation of the intrinsic phase. Computing the inverse Fourier transform of the HHG spectra above the 10th harmonic order, we can retrieve the attosecond pulse trains shown in purple in Figs. 2(d) and 2(e), for the outer and inner rings, respectively. The polarization state of the attosecond pulse trains can be monitored through the temporal evolution of the Stokes parameters [29]. The time-dependent ellipticity can be thus described as $\epsilon(t)=\tan \left[0.5 \arctan \left(S_{3}(t) / \sqrt{S_{1}^{2}(t)+S_{2}^{2}(t)}\right)\right]$, while the time-dependent tilt angle is $\theta(t)=0.5 \arctan \left[S_{2}(t) / S_{1}(t)\right]$.

In Figs. 2(d) and 2(e), we show the time-dependent ellipticity, $\epsilon(t)$, in green and the time-dependent tilt, $\theta(t)$, in orange for the outer and inner rings, together with the attosecond pulse train envelope in purple-which corresponds to the Stokes parameter $S_{0}(t)$. We can see how the polarization state of the attosecond pulses evolves from LCP (RCP) to RCP (LCP) along the train for the outer (inner) spatial ring. The tilt angle exhibits a rotation from pulse to pulse and also within each individual pulse. When driven by a single bichromatic counterrotating field, the tilt angle is known to rotate 120 deg from pulse to pulse. In contrast, in this scenario, the rotation is more complicated due to the overlap of the two time-delayed fields. The structure of the trains remains almost invariant within a divergence angle width of $0.5 \mathrm{mrad}$, approximately. Finally, we note that the time-dependent degree of polarization, defined as $P(t)=\sqrt{S_{1}^{2}(t)+S_{2}^{2}(t)+S_{3}^{2}(t)} / S_{0}(t)$, is constant and equal to one for all of the cases presented in this work, as expected from the coherence of the HHG process.

To have a better insight into the origin of the temporal structure of the attosecond pulse trains, we present in Fig. 3 the time-frequency analysis for the two cases depicted in Fig. 2. At the outer ring (left column), the LCP harmonics dominate at the front part of the pulse train, whereas RCP harmonics dominate at the rear part of the pulse. This behavior is reversed at the inner ring (right column). Note that the temporal delay between the OAM bi-circular drivers $A$ and $B$ is chosen to be equal to the FWHM of the driving pulses in order to obtain a homogeneous and linear variation of the ellipticity over time. A different choice of the time delay can be used to customize the temporal variation of the ellipticity of the attosecond pulse train. Analogously, the choice of the driving pulse lengths, and their OAM, allows for a custom control of the time-dependent polarization structure of the attosecond pulse train. To show the versatility of this technique, we have performed simulations with few-cycle driving laser pulses and a different OAM combination. In Fig. 4, we present the simulation results when considering Laguerre-Gauss beams with $\ell_{1}^{A}=2, \ell_{2}^{A}=-1$ (and, thus, $\ell_{1}^{B}=1, \ell_{2}^{B}=-2$ ), all of them with $p=0$. The driving pulse length is $1.9 \mathrm{fs}$ FWHM, and the temporal delay between the $A$ and $B$ drivers is $t_{d}=1.9 \mathrm{fs}$. The waist of the driving beams is $w_{0}=30 \mu \mathrm{m}$ for $\ell= \pm 1$ and $w_{0}=21.4 \mu \mathrm{m}$ for $\ell= \pm 2$. Now, the number of attosecond pulses in the train is reduced-as expected-, and, thus, the variation rate of the ellipticity in time increases. In this case, we present the attosecond pulse train emitted at an intermediate divergence angle (detected at $1.7 \mathrm{mrad}$ ) in order to illustrate the possibility of obtaining a polarization evolution from linear to highly elliptical, in contrast to the elliptical-to-elliptical polarization variation in the outer and inner rings. On the other hand, the

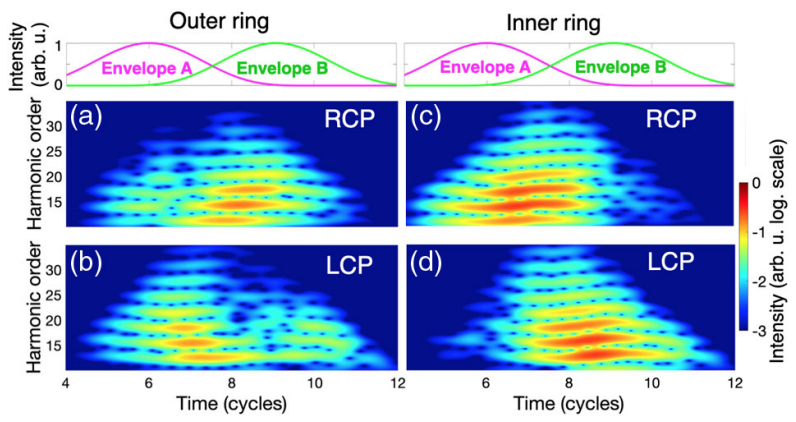

Fig. 3. Time-frequency analysis for RCP (first row) and LCP (second row) projections of the harmonic emission at the outer (left column) and inner (right column) rings, depicted in Fig. 2. The outer (inner) ring LCP (RCP) harmonics precede temporally the RCP (LCP) ones. The spectral width of the Gaussian mask used to perform the time-frequency analysis is $\omega_{1}$. 


\section{Few-cycle laser pulses}
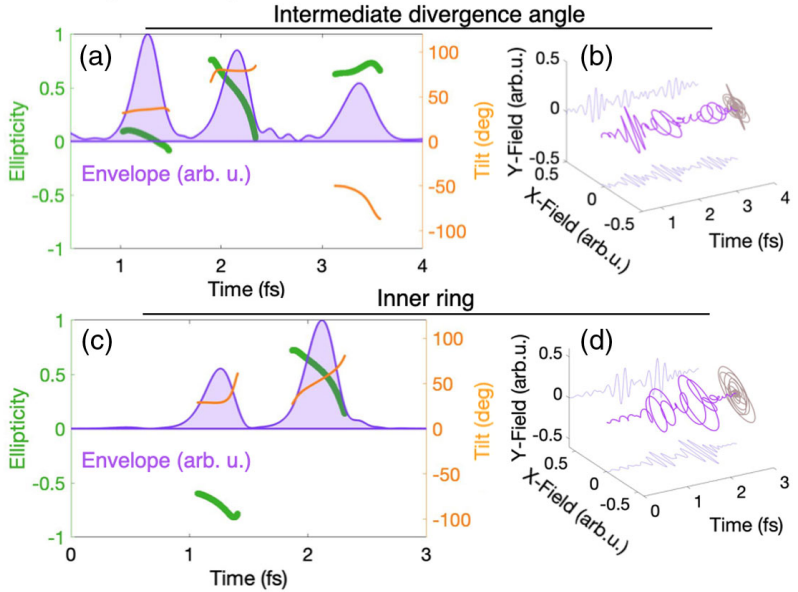

Fig. 4. Attosecond pulses-envelope (purple), ellipticity (green), and tilt angle (orange) - obtained with a few-cycle OAM bi-circular field with $\ell_{1}^{A}=2, \ell_{2}^{A}=-1$ (and, thus, $\ell_{1}^{B}=1, \ell_{2}^{B}=-2$ ) for an intermediate divergence angle (top) and inner ring (bottom) emission. The two-dimensional (2D) evolution of the attosecond electric fields is depicted in the right column.

inner ring consists of two elliptically polarized attosecond pulses with opposite helicities (detected at $1 \mathrm{mrad}$ ).

In conclusion, we have presented a scheme for the generation of attosecond pulse trains with time-ordered polarization states. By driving HHG with two time-delayed OAM bi-circular drivers, two spatially separated rings of harmonics are generated, each with polarization evolving from LCP to RCP and vice versa. The proper choice of the OAM, pulse length, and time delay of the driving pulses allows for custom control over the temporal evolution of the SAM of the pulses within the train.

We, thus, introduce a novel structured ultrafast light source by adding a new degree of freedom, time-ordered SAM, or polarization into the quantum stroboscope scheme. Similarly, time-ordered OAM-or self-torque-was introduced recently into the family of structured light beams thanks to HHG [26]. By adding this temporal dependency into the SAM of attosecond EUV beams, this work opens the route towards new probing scenarios-such as the study of ultrafast dynamics in chiral systems, pump-probe experiments with polarization varying states, or time-dependent polarization $\mathrm{x}$-ray magnetic circular dichroism - that provide an alternative route to probe the ultrafast dynamics of matter systems at the nanoscale.

Funding. Junta de Castilla y León (SA287P18); FEDER funds; Ministerio de Ciencia, Innovación y Universidades (FIS2016-75652-P, RYC-2017-22745, PID2019-106910GBI00); Ministerio de Educación, Cultura y Deporte (FPU16/02591); European Research Council (ERC) (851201); Barcelona Supercomputing Center (FI-2019-1-0013).

Disclosures. The authors declare no conflicts of interest.

\section{REFERENCES}

1. A. McPherson, G. Gibson, H. Jara, U. Johann, T. S. Luk, I. A. McIntyre, K. Boyer, and C. K. Rhodes, J. Opt. Soc. Am. B 4, 595 (1987).
2. M. Ferray, A. L'Huillier, X. F. Li, L. A. Lompre, G. Mainfray, and C. Manus, J. Phys. B 21, L31 (1988).

3. G. Farkas and C. Tóth, Phys. Lett. A 168, 447 (1992).

4. P. M. Paul, E. S. Toma, P. Breger, G. Mullot, F. Augé, P. Balcou, H. G. Muller, and P. Agostini, Science 292, 1689 (2001).

5. M. Hentschel, R. Kienberger, C. Spielmann, G. A. Reider, N. Milosevic, T. Brabec, P. Corkum, U. Heinzmann, M. Drescher, and F. Krausz, Nature 414, 509 (2001).

6. J. Mauritsson, P. Johnsson, E. Mansten, M. Swoboda, T. Ruchon, A. L'Huillier, and K. J. Schafer, Phys. Rev. Lett. 100, 073003 (2008).

7. K. J. Schafer, B. Yang, L. F. DiMauro, and K. C. Kulander, Phys. Rev. Lett. 70, 1599 (1993).

8. P. Dietrich, N. H. Burnett, M. Ivanov, and P. B. Corkum, Phys. Rev. A 50, R3585 (1994).

9. P. Huang, C. Hernández-García, J. Huang, P. Huang, L. Rego, C. Lu, S. Yang, L. Plaja, A. H. Kung, and M. Chen, IEEE J. Sel. Top. Quantum Electron. 25, 1 (2019).

10. A. Ferré, C. Handschin, M. Dumergue, F. Burgy, A. Comby, D. Descamps, B. Fabre, G. A. Garcia, R. Géneaux, L. Merceron, E. Mével, L. Nahon, S. Petit, B. Pons, D. Staedter, S. Weber, T. Ruchon, V. Blanchet, and Y. Mairesse, Nat. Photonics 9, 93 (2015).

11. Ó. Zurrón-Cifuentes, R. Boyero-García, C. Hernández-García, A. Picón, and L. Plaja, Opt. Express 27, 7776 (2019).

12. G. Lambert, B. Vodungbo, J. Gautier, B. Mahieu, V. Malka, S. Sebban, P. Zeitoun, J. Luning, J. Perron, A. Andreev, S. Stremoukhov, F. Ardana-Lamas, A. Dax, C. P. Hauri, A. Sardinha, and M. Fajardo, Nat. Commun. 6, 6167 (2015).

13. D. D. Hickstein, F. J. Dollar, P. Grychtol, J. L. Ellis, R. Knut, C. Hernandez-Garcia, D. Zusin, C. Gentry, J. M. Shaw, T. Fan, K. M. Dorney, A. Becker, A. Jaron-Becker, H. C. Kapteyn, M. M. Murnane, and C. G. Durfee, Nat. Photonics 9, 743 (2015).

14. C. Hernández-García, C. G. Durfee, D. D. Hickstein, T. Popmintchev, A. Meier, M. M. Murnane, H. C. Kapteyn, I. J. Sola, A. Jaron-Becker, and A. Becker, Phys. Rev. A 93, 043855 (2016).

15. P.-C. Huang, C. Hernández-García, J.-T. Huang, P.-Y. Huang, C.-H. Lu, L. Rego, D. D. Hickstein, J. L. Ellis, A. Jaron-Becker, A. Becker, S.-D. Yang, C. G. Durfee, L. Plaja, H. C. Kapteyn, M. M. Murnane, A. H. Kung, and M.-C. Chen, Nat. Photonics 12, 349 (2018).

16. J. L. Ellis, K. M. Dorney, D. D. Hickstein, N. J. Brooks, C. Gentry, C. Hernández-García, D. Zusin, J. M. Shaw, Q. L. Nguyen, C. A. Mancuso, G. S. Matthijs Jansen, S. Witte, H. C. Kapteyn, and M. M. Murnane, Optica 5, 479 (2018).

17. D. Azoury, O. Kneller, M. Krüger, B. D. Bruner, O. Cohen, Y. Mairesse, and N. Dudovich, Nat. Photonics 13, 198 (2019).

18. H. Eichmann, A. Egbert, S. Nolte, C. Momma, B. Wellegehausen, W. Becker, S. Long, and J. K. Mclver, Phys. Rev. A 51, R3414 (1995).

19. S. Long, W. Becker, and J. K. Mclver, Phys. Rev. A 52, 2262 (1995).

20. D. B. Milošević, W. Becker, and R. Kopold, Phys. Rev. A 61, 063403 (2000).

21. A. D. Bandrauk, J. Guo, and K. J. Yuan, J. Opt. 19, 124016 (2017).

22. A. Fleischer, O. Kfir, T. Diskin, P. Sidorenko, and O. Cohen, Nat. Photonics 8, 543 (2014).

23. O. Kfir, P. Grychtol, E. Turgut, R. Knut, D. Zusin, D. Popmintchev, T. Popmintchev, H. Nembach, J. M. Shaw, A. Fleischer, H. Kapteyn, M. Murnane, and O. Cohen, Nat. Photonics 9, 99 (2015).

24. K. M. Dorney, L. Rego, N. J. Brooks, J. San Román, C.-T. Liao, J. L. Ellis, D. Zusin, C. Gentry, Q. L. Nguyen, J. M. Shaw, A. Picón, L. Plaja, H. C. Kapteyn, M. M. Murnane, and C. Hernández-García, Nat. Photonics 13, 123 (2018).

25. L. Barreau, K. Veyrinas, V. Gruson, S. J. Weber, T. Auguste, J.-F. Hergott, F. Lepetit, B. Carré, J.-C. Houver, D. Dowek, and P. Salières, Nat. Commun. 9, 4727 (2018).

26. L. Rego, K. M. Dorney, N. J. Brooks, Q. L. Nguyen, C.-T. Liao, J. San Román, D. E. Couch, A. Liu, E. Pisanty, M. Lewenstein, L. Plaja, H. C. Kapteyn, M. M. Murnane, and C. Hernández-García, Science 364, eaaw9486 (2019).

27. I. J. Sola, E. Mével, L. Elouga, E. Constant, V. Strelkov, L. Poletto, P. Villoresi, E. Benedetti, J. P. Caumes, S. Stagira, C. Vozzi, G. Sansone, and M. Nisoli, Nat. Phys. 2, 319 (2006).

28. C. Hernández-García, J. A. Pérez-Hernández, J. Ramos, E. C. Jarque, L. Roso, and L. Plaja, Phys. Rev. A 82, 033432 (2010).

29. W. H. McMaster, Am. J. Phys. 22, 351 (1954). 